\title{
Economic inequality and mobility in kinetic models for social sciences
}

\author{
Maria Letizia Bertotti ${ }^{1}$, Giovanni Modanese ${ }^{2}$ \\ ${ }^{1}$ Faculty of Science and Technology, Free University of Bozen-Bolzano, Italy, marialetizia.bertotti@ unibz.it \\ ${ }^{2}$ Faculty of Science and Technology, Free University of Bozen-Bolzano, Italy, giovanni.modanese@ unibz.it
}

\begin{abstract}
Statistical evaluations of the economic mobility of a society are more difficult than measurements of the income distribution, because they require to follow the evolution of the individuals' income for at least one or two generations. In micro-to-macro theoretical models of economic exchanges based on kinetic equations, the income distribution depends only on the asymptotic equilibrium solutions, while mobility estimates also involve the detailed structure of the transition probabilities of the model, and are thus an important tool for assessing its validity. Empirical data show a remarkably general negative correlation between economic inequality and mobility, whose explanation is still unclear. It is therefore particularly interesting to study this correlation in analytical models. In previous work we investigated the behavior of the Gini inequality index in kinetic models in dependence on several parameters which define the binary interactions and the taxation and redistribution processes: saving propensity, taxation rates gap, tax evasion rate, welfare means-testing etc. Here, we check the correlation of mobility with inequality by analyzing the mobility dependence from the same parameters. According to several numerical solutions, the correlation is confirmed to be negative.

Keywords: kinetic models, taxation and redistribution, economic inequality, social mobility
\end{abstract}

\section{Introduction}

The rise of economic inequality has become, in recent times, cause for serious concern and the object of frequent debates. Inequality is increasing in several countries and also appears to be accompanied by a diminution of social mobility. This combination is especially harmful, because it frustrates the attempts of many individuals to move up the income ladder and improve their situation. Empirical surveys confirm the existence of a negative correlation between mobility and inequality [1,2]. It seems therefore very important to highlight the fundamental causes of this correlation.

Our aim in this paper is to explore these themes through mathematical methods, by providing a suitable definition of mobility and by comparing the possible scenarios compatible with different policies and conditions. In this connection, we introduce some indicators apt to measure various aspects of mobility within a family of kinetic-type models for the description of economic exchanges in a closed society. We investigate the relationship between economic inequality and mobility by analyzing income distributions emerging from different fiscal and welfare systems. The output of a large number of simulations shows clear evidence of a negative correlation between mobility and equality, in agreement with the qualitative features of real world situations. The models also allow to study the simultaneous dependence of the Gini index and the mobility parameters on taxation rates and welfare means-testing, and to track variations in the mobility of different income classes. In particular, it turns out (the simulations suggest) that there are, with reference to variables measuring welfare and taxation, lines on which both the Gini index and the mobility parameter are constant.

Our approach fits in with a complex system perspective, in the sense that we find the emergence of collective macro-level patterns out of a whole of interactions occurring at the micro level. The need of considering, when dealing with socio-economic questions, a multiplicity of individual interactions rather than a representative agent is highlighted and advocated e.g. in [3, 4, 5]. For this purpose, agent based computational models have been typically developed and applied within the economics community, [6]. On the other side, during the last two decades a number of physicists have started to tackle economic and financial questions by using tools of statistical physics, see e.g. [7, 8, 9] and the recent review [10]. The reason for that naturally arises from certain analogies existing between markets or populations of individuals who exchange money and particle systems or gases in which molecules collide exchanging energy. In particular, as illustrated below, our methods and models allow, thanks to their characteristic features, the definition and investigation of a mobility concept.

The remainder of the paper is organized as follows. Section 2 sets up a framework for the description of monetary exchanges in a closed society. In Section 3 a family of models is constructed within this framework. In the fourth 
section the concept of social mobility is shortly discussed and quantitative indicators to measure it are introduced. Hence, the correlation between the economic inequality and mobility is explored. Finally, a summary is contained in the last section.

\section{A discrete-type framework for the description of monetary exchanges in a closed society}

Moving from a stylized description of the complex of monetary exchanges which take place in a closed market society, we outline in this section a discrete-type framework suitable for its modelling. This framework was proposed and discussed in our previous works [11, 12, 13], but we give here a slightly different introduction. The problem at issue concerns the formation of the income distribution curve and the economic inequality in a population of constant size. If the variable $r \in[0,+\infty)$ denotes the individual income and $x(r)$ the density of the population having income $r$, one could say that the total population and the total income are given by $\int_{0}^{+\infty} x(r) d r$ and $\int_{0}^{+\infty} r x(r) d r$ respectively. However, it is quite natural and also convenient in view of a numerical treatment of the problem to divide the population into a finite number of classes. Toward this goal, we consider the $n+1$ numbers $0=r_{0}<r_{1}<r_{2}<\ldots<r_{n}$ with $r_{n}$ so large as to represent a sort of supremum of the possible income of any individual in the given society. For simplicity we assume the density of the population whose income belongs to the interval $\left[r_{i-1}, r_{i}\right)$ to be constant and denote it by $x_{i}$ for $i=1, \ldots, n$. The fraction of individuals having income in $\left[r_{i-1}, r_{i}\right)$ is then

$$
X_{i}=\int_{r_{i-1}}^{r_{i}} x_{i} d r=x_{i}\left(r_{i}-r_{i-1}\right)
$$

and its income is

$$
R_{i}=\int_{r_{i-1}}^{r_{i}} r x_{i} d r=x_{i} \frac{\left(r_{i}^{2}-r_{i-1}^{2}\right)}{2} .
$$

We refer to the class of individuals with income in $\left[r_{i-1}, r_{i}\right)$ as to the $i$-th class and to its individuals as to $i$-individuals. An immediate consequence of (1) and (2) is that $R_{i}=X_{i} \tilde{r}_{i}$, where $\tilde{r}_{i}=\left(r_{i-1}+r_{i}\right) / 2$ denotes the average income of individuals in the $i$-th class.

We assume that pairwise monetary exchanges take place between single individuals. A "microscopic" description of this can be as follows: when an $h$-individual pays to a $k$-individual an amount of money $S$ (with $S<\left(\tilde{r}_{i+1}-\tilde{r}_{i}\right)$ for all $i=1, \ldots, n)$, the $h$-th class gets slightly poorer, while the $k$-th class gets slightly richer. This induces a retrocession of a portion of the population of the $h$-th to the $(h-1)$-th class and an advance of a portion of the $k$-th population to the $(k+1)$-th class. Easy calculations show that, if the total population and the total income have to remain unchanged as happens in the conservative case dealt with here, then the variations of population in the $h$-th and in the $k$-th class are given by

$$
\Delta X_{h}=-\frac{2 S}{r_{h}-r_{h-2}} \quad \text { and } \quad \Delta X_{k}=-\frac{2 S}{r_{k+1}-r_{k-1}} .
$$

How often monetary exchanges can occur can be postulated through the definition of suitable terms $p_{h, k}$ for $h, k=$ $1, \ldots, n$, with each $p_{h, k}$ accounting for the encounter frequency rate of individuals of the $h$-th and in the $k$-th class and expressing as well the probability that in an encounter between an $h$-individual and a $k$-individual, the one who pays is the $h$-individual. The possibility that none of the two pays has to be taken into account as well. Hence, for any $h$ and $k$ in $\{1, \ldots, n\}$, the inequalities $0 \leq p_{h, k} \leq 1$ and $p_{h, k}+p_{k, h} \leq 1$ have to hold true. Apart from that, the values of the $p_{h, k}$ should be determined based on phenomenological observations. A specific choice for the values of the $p_{h, k}$ will be done in the third section of this note.

Beside direct money exchanges, we also assume the existence of a process of taxation and redistribution. If we imagine that the money received form the $k$-individual is subject to taxation with a tax rate equal to $\tau_{k} \in[0,1)$, supposing (at least as a first case study) that the $k$-individual pays the due tax, we observe that the entire operation is equivalent to one in which the $h$-individual pays a quantity $S$, the $k$-individual earns a quantity $S\left(1-\tau_{k}\right)$, and the quantity $S \tau_{k}$ goes to the government. We then imagine, here again postulating an irreproachable situation, that the government redistributes the tax revenue (which is just $S \tau_{k}$ for the single mentioned interaction) to the entire population through the provision of welfare services: education, health assistance and so on. At this step, we observe that in fact the government intervention can be ignored, since the mechanism can be equivalently described as follows: in correspondence to the mentioned direct interaction between an $h$-individual and a $k$-individual, individuals of all 
classes receive an amount of money corresponding to a returned tax portion. In other words, the taxation and redistribution effect is equivalent to the effect of an indirect interaction between the paying $h$-individual and all others $\|^{1}$ Of course, the indirect interactions too contribute to the possibility of class change of individuals.

In order to formalize the overall mechanism described so far we introduce

- the direct transition probabilities $C_{h k}^{i} \in[0,1]$ for $i, h, k \in\{1, \ldots, n\}$, satisfying

$$
\sum_{i=1}^{n} C_{h k}^{i}=1 \quad \text { for any } h, k \in\{1, \ldots, n\}
$$

where $C_{h k}^{i}$ expresses the probability that an individual of the $h$-th class will belong to the $i$-th class after a direct interaction with an individual of the $k$-th class,

and

- the indirect transition variations $T_{[h k]}^{i}: \mathbf{R}^{n} \rightarrow \mathbf{R}$ for $i, h, k \in\{1, \ldots, n\}$, where each $T_{[h k]}^{i}(X)$ with $X=\left(X_{1}, \ldots, X_{n}\right) \in$ $\mathbf{R}^{n}$ is a continuous function, satisfying

$$
\sum_{i=1}^{n} T_{[h]}^{i}(X)=0 \quad \text { for any } h, k \in\{1, \ldots, n\} \text { and } X \in \mathbf{R}^{n},
$$

accounting for the indirect interactions and expresses the variation in the $i$-th class due to an interaction between an $h$-individual and a $k$-individual.

As will be shown in the following section, the construction of both the parameters $C_{h k}^{i}$ and the functions $T_{[h k]}^{i}$ is accomplished combining the variation terms 3 and the probabilities $p_{h, k}$.

The variation in time of the number of individuals belonging to the $n$ income classes is described by the following balance differential equations:

$$
\frac{d X_{i}}{d t}=\sum_{h=1}^{n} \sum_{k=1}^{n} C_{h k}^{i} X_{h} X_{k}-\sum_{h=1}^{n} \sum_{k=1}^{n} C_{i k}^{h} X_{i} X_{k}+\sum_{h=1}^{n} \sum_{k=1}^{n} T_{[h k]}^{i}(X) X_{h} X_{k} \quad \text { for } i \in\{1, \ldots, n\},
$$

which, in view of (4), can be simplified to take the form:

$$
\frac{d X_{i}}{d t}=\sum_{h=1}^{n} \sum_{k=1}^{n}\left(C_{h k}^{i}+T_{[h k]}^{i}(X)\right) X_{h} X_{k}-X_{i} \sum_{k=1}^{n} X_{k} \quad \text { for } i \in\{1, \ldots, n\} .
$$

They are nonlinear ordinary differential equations.

\section{An example of model family}

In order to construct a specific model or model family, we start by fixing specific values for the $p_{h, k}$. We take them as

$$
p_{h, k}=\min \left\{\tilde{r}_{h}, \tilde{r}_{k}\right\} / 4 \tilde{r}_{n}
$$

with the exception of the terms $p_{j, j}=\tilde{r}_{j} / 2 \tilde{r}_{n}$ for $j=2, \ldots, n-1, p_{h, 1}=\tilde{r}_{1} / 2 \tilde{r}_{n}$ for $h=2, \ldots, n, p_{n, k}=\tilde{r}_{k} / 2 \tilde{r}_{n}$ for $k=1, \ldots, n-1, p_{1, k}=0$ for $k=1, \ldots, n$ and $p_{h n}=0$ for $h=1, \ldots, n$. This choice is suggested by the consideration that poorer individuals typically earn and pay smaller amounts than richer ones and do so less frequently. It guarantees a degree of heterogeneity among people of different classes, also in connection with their interactions with others.

\footnotetext{
${ }^{1}$ We point out that actually, for a technical reason, in the following we will assume that no redistribution goes to individuals of the $n$-th class. Indeed, if an individual of the $n$-th class would receive some money, the possibility would arise for him to advance to a higher class, which is impossible.
} 
Recalling now the formulae 3 , we assume that the only possibly nonzero elements among the $C_{h k}^{i}$ are:

$$
\begin{aligned}
C_{i+1, k}^{i} & =p_{i+1, k} \frac{2 S\left(1-\tau_{k}\right)}{r_{i+1}-r_{i-1}}, \\
C_{i, k}^{i} & =1-p_{k, i} \frac{2 S\left(1-\tau_{i}\right)}{r_{i+1}-r_{i-1}}-p_{i, k} \frac{2 S\left(1-\tau_{k}\right)}{r_{i}-r_{i-2}}, \\
C_{i-1, k}^{i} & =p_{k, i-1} \frac{2 S\left(1-\tau_{i-1}\right)}{r_{i}-r_{i-2}} .
\end{aligned}
$$

We stress that the expression for $C_{i+1, k}^{i}$ in 8 holds true for $i \leq n-1$ and $k \leq n-1$; the second addendum of the expression for $C_{i, k}^{i}$ is effectively present only provided $i \leq n-1$ and $k \geq 2$, while its third addendum is present only provided $i \geq 2$ and $k \leq n-1$; the expression for $C_{i-1, k}^{i}$ holds true for $i \geq 2$ and $k \geq 2$.

As for the $T_{[h k]}^{i}$, we first introduce certain coefficients $w_{i}$ for $i=1, \ldots, n$, which denote the weights of a differently distributed welfare. In the following we will take the $r_{j}$ (and consequently the $\tilde{r}_{j}$ ) linear in $j: r_{j}=c j$ for some constant $c>0$. Then, a conceivable expression for the weights $w_{i}$, according to which, the $w_{j}$ decrease linearly as functions of $j$, is given by

$$
w_{j}=\tilde{r}_{n+1-j}+\frac{2}{n-1} \gamma\left(j-\frac{n+1}{2}\right)\left(\tilde{r}_{n}-\tilde{r}_{1}\right),
$$

with $\gamma \in(0,1 / 2)$. The smaller $\gamma \in(0,1 / 2)$, the larger is the difference $w_{1}-w_{n}$. Indeed, $w_{1}-w_{n}=\left(\tilde{r}_{n}-\tilde{r}_{1}\right)(1-2 \gamma)$. The value $\gamma=1 / 2$ also is admitted, but if $\gamma=1 / 2$, then $w_{j}$ has the same value for each $j=1, \ldots, n$.

We can now express the $T_{[h k]}^{i}$ as

$$
T_{[h k]}^{i}(X)=U_{[h k]}^{i}(X)+V_{[h k]}^{i}(X)
$$

where 2

$$
U_{[h k]}^{i}(X)=\frac{p_{h, k} 2 S \tau_{k}}{\sum_{j=1}^{n} w_{j} X_{j}}\left(\frac{w_{i-1} X_{i-1}}{r_{i}-r_{i-2}}-\frac{w_{i} X_{i}}{r_{i+1}-r_{i-1}}\right)
$$

represents the variation corresponding to the advancement from a class to the subsequent one, due to the benefit of taxation and 3

$$
V_{[h k]}^{i}(X)=p_{h, k} 2 S \tau_{k} \frac{\sum_{j=1}^{n-1} w_{j} X_{j}}{\sum_{j=1}^{n} w_{j} X_{j}}\left(\frac{\delta_{h, i+1}}{r_{h}-r_{i-1}}-\frac{\delta_{h, i}}{r_{h}-r_{i-2}}\right),
$$

with $\delta_{h, k}$ denoting the Kronecker delta, represents the variation corresponding to the retrocession from a class to the preceding one, due to the payment of some tax.

We notice here that the effective amount of money representing taxes, which is paid in correspondence to a payment of $S\left(1-\tau_{k}\right)$ and is then redistributed is $S \tau_{k}\left(\sum_{j=1}^{n-1} w_{j} X_{j}\right) /\left(\sum_{j=1}^{n} w_{j} X_{j}\right)$ instead of $S \tau_{k}$. This is in agreement with the fact that, due to the bound on the admissible income, individuals of the $n$-th class do not benefit from the redistribution (see Note 1).

Finally, to fix ideas, we take $S=1$,

$$
r_{j}=25 j,
$$

and

$$
\tau_{j}=\tau_{\min }+\frac{j-1}{n-1}\left(\tau_{\max }-\tau_{\min }\right)
$$

for $j=1, \ldots, n$, where $\tau_{\min }$ and $\tau_{\max }$ respectively denote the minimum and maximum tax rate. Still, the value of $\gamma, \tau_{\min }$ and $\tau_{\max }$ have to be fixed. Hence, the equations 7 describe a family of models rather than a single model.

Finding analytical solutions for these equations is in practice hopeless. But, as will be shown in the next section, several relevant facts can be understood through simulations. Before passing to numerical results, we would like to

\footnotetext{
${ }^{2}$ In $[11, h>1$ and the terms involving the index $i-1$ [respectively, $i+1$ ] are effectively present only provided $i-1 \geq 1$ [respectively, $i+1 \leq n$ ]. In other words: the $1^{o}$ term into parentheses on the r.h.s. of 11 is present for $2 \leq i \leq n$; the $2^{o}$ term into parentheses on the r.h.s. of 11 is present for $1 \leq i \leq n-1$.

${ }^{3}$ In $[12$, $h>1$ and the terms involving the index $i-1$ [respectively, $i+1$ ] are effectively present only provided $i-1 \geq 1$ [respectively, $i+1 \leq n$ ]. In other words: the $1^{o}$ term into parentheses on the r.h.s. of [12) is present for $1 \leq i \leq n-1$; the $2^{o}$ term into parentheses on the r.h.s. of (12) is present for $2 \leq i \leq n$.
} 
mention the following two properties of (7). For both of them the proof of the corresponding result established in [11] and referring in fact to a slightly different model can be easily adapted.

Well posedness of the Cauchy problem. For fixed values of the parameters $\gamma, \tau_{\min }$ and $\tau_{\max }$, in correspondence to any initial condition $X_{0}=\left(X_{01}, \ldots, X_{0 n}\right)$, for which $X_{0 i} \geq 0$ for all $i=1, \ldots, n$ and $\sum_{i=1}^{n} X_{0 i}=1$, a unique solution $X(t)=\left(X_{1}(t), \ldots, X_{n}(t)\right)$ of (7) exists, which is defined for all $t \in[0,+\infty)$, satisfies $X(0)=X_{0}$ and also

$$
X_{i}(t) \geq 0 \quad \text { for } i=1, \ldots, n \quad \text { and } \quad \sum_{i=1}^{n} X_{i}(t)=1 \quad \text { for all } t \geq 0 .
$$

Conservation of the total income $\mu$. For fixed values of the parameters $\gamma, \tau_{\min }$ and $\tau_{\max }$, the scalar function $\mu(X)=$ $\sum_{i=1}^{n} \tilde{r}_{i} X_{i}$, expressing the total (and mean) income, is a first integral for the system (7).

From now on, we report on the outputs of a large number of simulations. To run them, we fixed $n=15$. The first fact one observes is the following one.

Uniqueness, for any fixed value of $\mu$, of the asymptotic stationary distribution. For fixed values of the parameters $\gamma, \tau_{\min }$ and $\tau_{\max }$, for any fixed value $\mu \in\left[\tilde{r}_{1}, \tilde{r}_{n}\right]$, an equilibrium of (7) exists, to which all solutions of (7), whose initial conditions $X_{0}=\left(X_{01}, \ldots, X_{0 n}\right)$ satisfy $X_{0 i} \geq 0$ for all $i=1, \ldots, n, \sum_{i=1}^{n} X_{0 i}=1$, and $\sum_{i=1}^{n} \tilde{r}_{i} X_{0 i}=\mu$ tend asymptotically as $t \rightarrow+\infty$. In other words, a one-parameter family of asymptotic stationary distributions exists, the parameter being the total income value.

Other properties can be established from comparisons between different models of the family under consideration. In previous work ([14, 15]) we investigated the effects on the shape of the asymptotic income distribution curve and the economic inequality produced by the adoption of fiscal systems with different spread between the maximum and the minimum tax rates and also produced by the introduction of differently weighted welfare measures. Briefly, the simulations suggested the following properties.

Dependence of the asymptotic stationary distribution on the tax rate difference $\tau_{\max }-\tau_{\min }$. The profile of the asymptotic stationary distribution depends on the difference between the maximum and the minimum tax rate: if $\tau_{\max }-\tau_{\min }$ is enlarged, while all other data are kept unchanged, then an increase of the fraction of individuals belonging to the middle classes (to the detriment of those in the poorest and richest classes) can be detected at the asymptotic equilibrium.

Dependence of the asymptotic stationary distribution on differently weighted welfare measures. The profile of the asymptotic stationary distribution depends on the parameter $\gamma$ characterising the welfare measures. When $\gamma$ decreases and all other data are kept unchanged, at the asymptotic equilibrium an increase of the fraction of individuals belonging to the middle classes can be detected (and, correspondingly, a decrease of those in the poorest and richest classes).

In this connection we evaluated for the models under consideration the Gini index $G$ and the tax revenue $T R$, whose definition is recalled next. Let the Lorenz curve represent (on the $y$ axis) the cumulative percentage of the total income of a population earned by the bottom percentage of individuals (on the $x$ axis). The Gini index $G$ corresponds to the ratio $A_{1} / A_{2}$ of the area $A_{1}$ between the line of perfect equality $y=x$ and the Lorenz curve and the total area $A_{2}$ under the line of perfect equality. It takes values in $[0,1], 0$ representing complete equality and 1 maximal inequality. The tax revenue is the total amount of tax collected in the unit time and redistributed as welfare provisions:

$$
T R=\sum_{h=1}^{n} \sum_{k=1}^{n} \sum_{j=1}^{n-1} p_{h k} S \tau_{k} \frac{w_{j} \tilde{X}_{j}}{\left(\sum_{i=1}^{n} w_{i} \tilde{X}_{i}\right)} \tilde{X}_{h} \tilde{X}_{k}
$$

with $\tilde{X}_{i}$ denoting the fraction of individuals in the $i$-th class at equilibrium.

\section{Social mobility and its correlation with economic inequality}

This paper focuses upon an important socio-economic issue: social mobility 4

\footnotetext{
${ }^{4}$ The expressions social mobility and economic mobility are used here to designate the same concept.
} 


\subsection{Introductive remarks}

Social mobility gives a measure of the chances individuals have to improve their economic status through education and work. It is known that a high level of inequality in a society is especially harmful when it is complemented by a diminution of social mobility, see e.g. [1, 2]. Social mobility has been historically an important component of the "American Dream" (and of its emulations), thus making the high inequality level present in the US more tolerable. Today, several observers point out a diminution of social mobility, as seen for instance from the growing difficulties that the sons and daughters of the poor and middle classes have to access the best universities and the best paid jobs. As mentioned in the introduction, quantitative empirical surveys show that mobility is negatively correlated with inequality, i.e. more unequal societies also tend to have less mobility.

Studying the correlation between economic inequality and mobility is of particular interest because statistical evaluations of the economic mobility of a society are more difficult than measurements of the income distribution. This is due to the fact that they require to follow the evolution of the individuals' income for at least one or two generations. Correspondingly, in micro-to-macro theoretical models of economic exchanges based on kinetic equations, the income distribution depends only on the asymptotic equilibrium solution of the equations, while mobility estimates also involve the detailed structure of the transition probabilities of the model.

In our models there are no generations and it is impossible to compare the income of one individual with that of his or her parents. Individuals are supposed to live and work indefinitely, or at least for the time it takes for the system to reach its dynamical equilibrium. During this time the income distribution evolves from some initial conditions to a stationary state, in correspondence to which the number of individuals belonging to a certain class remains constant in time: at equilibrium, the total rate of individuals leaving that class is equal in absolute value to the total rate of individuals arriving from other classes. This means that in the equations (7), whose right hand side can now be written in a simplified form in view of $\left[15\right.$, one has $\dot{X}_{i}=0$. Equivalently, the equilibrium populations are solutions of the nonlinear equations

$$
\sum_{h=1}^{n} \sum_{k=1}^{n}\left(C_{h k}^{i}+T_{[h k]}^{i}(X)\right) X_{h} X_{k}-X_{i}=0 \quad \text { for } i \in\{1, \ldots, n\},
$$

with $C_{h k}^{i}$ and $T_{[h k]}^{i}(X)$ as in 8 and 10 .

The equilibrium populations can be obtained numerically as asymptotic solutions of the full evolution equations. We have evidence from several numerical solutions that the approach to equilibrium is exponential in time, in the sense that the "Cauchy convergence norm" converges exponentially to zero as $t \rightarrow \infty$ (see [14]).

Below we introduce quantitative indicators suitable to define the mobility in our models. Then, we check the correlation of mobility with inequality. To this end, we analyze the dependence of mobility on parameters such as the taxation rates gap and welfare means-testing; we find interesting relations between the mobility and the inequality indicators.

\subsection{Mobility in our model: detailed calculation and dependence on the model parameters}

The mobility evaluated in this paper is always meant at equilibrium, when the rates of the individuals entering and leaving an income class are the same (but of course differ from class to class). We do not consider long-term trends, characterized by an increase or diminution of the total income of the society. In our models the social mobility is essentially a short-term mobility, which gives each individual a probability to go up or down the income ladder. A large value of the mobility should be regarded as positive for the economy, because it acts as a stimulus and as a reward for the individuals who are more capable and hard-working. While in the average the upward mobility and downward mobility are the same at equilibrium, we can imagine that those who go up are economically stronger than those who go down. This might be evidenced, in an improved version of the model, by introducing some heterogeneity factors, and so labelling the individuals not only with their income class $i$ but possibly also with some "fitness" index related to education, number of economic links, etc.

To start with, we define the probabilities for an individual belonging to the income class $i$, for $i=2, \ldots, n-1$, to be promoted to the upper class following a direct interaction or an indirect interaction (i.e., through welfare provisions) respectively as

$$
P_{i, \text { exchanges }(\text { individual })}=\frac{S}{r_{i+1}-r_{i}} \sum_{k=1}^{n} p_{k, i}\left(1-\tau_{i}\right) \tilde{X}_{k}
$$


and

$$
P_{i, \text { welfare(individual })}=\frac{S}{r_{i+1}-r_{i}} \frac{w_{i}}{\sum_{j=1}^{n} w_{j} \tilde{X}_{j}} \sum_{h=1}^{n} \sum_{k=1}^{n} p_{h, k} \tau_{k} \tilde{X}_{h} \tilde{X}_{k} .
$$

We restrict our attention to all classes but the first and the last one, in order to avoid possible boundary effects. By summing the probabilities 17 and 18 we obtain the total probability $P_{i \text { (individual })}$ for a single individual advancement

$$
P_{i(\text { individual })}=P_{i, \text { exchanges(individual })}+P_{i, \text { wel fare (individual })}
$$

which is the base for the calculations that follow.

We then define "averaged" class probabilities of being promoted to the upper class (the one-step transition probabilities for the class $i$ ) respectively due to direct or indirect interactions as

$$
P_{i, \text { exchanges }(\text { class })}=\frac{1}{\left(1-\tilde{X}_{1}-\tilde{X}_{n}\right)} \frac{S}{r_{i+1}-r_{i}} \sum_{k=1}^{n} p_{k, i}\left(1-\tau_{i}\right) \tilde{X}_{k} \tilde{X}_{i}
$$

and

$$
P_{i, \text { welfare(class })}=\frac{1}{\left(1-\tilde{X}_{1}-\tilde{X}_{n}\right)} \frac{S}{r_{i+1}-r_{i}} \frac{w_{i} \tilde{X}_{i}}{\sum_{j=1}^{n} w_{j} \tilde{X}_{j}} \sum_{h=1}^{n} \sum_{k=1}^{n} p_{h, k} \tau_{k} \tilde{X}_{h} \tilde{X}_{k}
$$

for $i=2, \ldots, n-1$.

We have first introduced similar quantities in our paper [14], where we computed the ratio between the term due to binary exchanges (the quadratic part of the evolution equation) and the term due to tax redistribution (the cubic part). This ratio gives a measure of the "liberalism" of the society. We found that with equally distributed welfare the ratio stays, quite surprisingly, almost constant when we pass from the middle classes to the rich classes, although one might expect welfare provisions to be irrelevant for the class advancement of the super-rich. Let us now call

$$
P_{i(\text { class })}=P_{i, \text { exchanges (class) }}+P_{i, \text { wel fare(class })} .
$$

the sum of the probabilities (19) and 20].

If we make an histogram of $P_{i(\text { individual })}$ in a typical situation with Gini index $G=0.368$, we see that it increases with income and attains its largest values in correspondence of the richest classes (see Fig. 1). We know, however, that in real world income distributions, the fifth quintile generally includes a consistent share of the classes, since the richest classes are scarcely populated and most of the population concentrates in the low- and middle-income classes. This also happens in our models provided the initial conditions are such that most of the wealth initially belongs to the low and middle classes. For instance, in the example with the parameters of Fig. 1, the histogram of mobility is shown in Fig. 2. So, while some aspects of the mobility of the rich are interesting in principle, because they are related to the formation of the Pareto fat tail in the distribution, the value of the mobility of the rich classes does not have a strong influence on the average mobility of all classes.

Our main measure of mobility expresses the collective probability of class advancement of all classes from the 2 -th to the $(n-1)$-th one. Indeed, we consider

$$
P_{\text {exchanges }(\text { collective })}=\frac{1}{\left(1-\tilde{X}_{1}-\tilde{X}_{n}\right)} \frac{S}{r_{i+1}-r_{i}} \sum_{i=2}^{n-1} \sum_{k=1}^{n} p_{k, i}\left(1-\tau_{i}\right) \tilde{X}_{k} \tilde{X}_{i}
$$

and

$$
P_{\text {welfare(collective })}=\frac{1}{\left(1-\tilde{X}_{1}-\tilde{X}_{n}\right)} \frac{S}{r_{i+1}-r_{i}} \sum_{i=2}^{n-1} \frac{w_{i} \tilde{X}_{i}}{\sum_{j=1}^{n} w_{j} \tilde{X}_{j}} \sum_{h=1}^{n} \sum_{k=1}^{n} p_{h, k} \tau_{k} \tilde{X}_{h} \tilde{X}_{k}
$$

and take the sum

$$
M=P_{\text {exchanges(collective })}+P_{\text {wel fare(collective) }}
$$

as the parameter which represents the mobility.

Now, we change slightly one of the parameters of the model, in order to increase equality and decrease the Gini index. For instance, we pass from taxation rates 30\%-45\% to 30\%-60\%, leaving unchanged the parameters defining the direct exchanges and the welfare redistribution parameter (here we have $\gamma=0.5$, which means uniform welfare distribution). This leads to a Gini coefficient $G=0.338$, to a variation $\Delta P_{i(\text { class })}$ as in Fig. 3 and to an average variation 


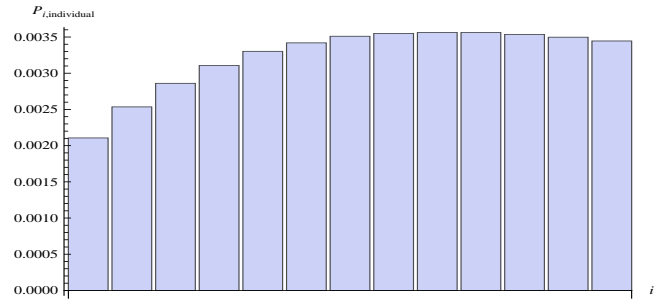

Figure 1: Individual probability of single-class advancement $P_{i \text { (individual) }}$ obtained from a numerical simulation with 15 income classes. The probability has been computed from the equilibrium population and from the coefficients $p_{i, k}$, $\tau_{i}$, according to Eq. (7), in a case where $\tau_{\min }=30 \%, \tau_{\max }=45 \%$ and the resulting Gini index is $G=0.368$. Note that the rich classes have the largest individual mobility. The initial conditions for the numerical simulation are such that most of the wealth initially belongs to the low and middle classes.

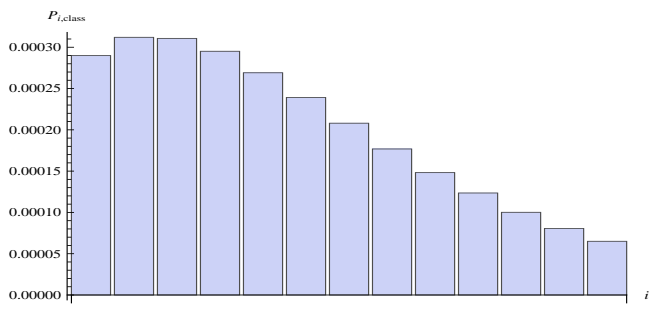

Figure 2: Histogram of the class mobility $P_{i(\text { class })}$ with the same parameters as in Fig. 1 . This is obtained from the histogram of the individual mobility, weighing each column with the normalized population of the corresponding class. Since the low and middle classes are much more populated than the rich classes (the first 7 or 8 classes typically comprise $4 / 5$ of the entire population), the weights affect strongly the relative contributions of the individual mobilities of Fig. 1 . The total mobility $M$ is determined by a weighted average.

$\Delta M=5.7 \cdot 10^{-5}$. It is clear from the histogram that the mobility variation for the middle classes is positive, and that it prevails in the average. The histogram of the individual mobility variations shows an even sharper diminution of the mobility of the rich classes, see Fig. 4. In fact, one could verify that also the percentage mobility variation is always larger for the rich classes, both for $P_{(\text {individual })}$ and $P_{(\text {class })}$. A similar behavior is found when the negative variation of the Gini index is obtained with a change of the welfare redistribution parameter, as to make it more favorable to the low and middle classes. Several trials of this kind confirm the positive correlation between equality and mobility. In the next section we shall consider variations of the Gini index and mobility in correspondence to contemporary variations in tax rates and welfare distribution parameters, which again confirm this positive correlation.

\subsection{Gini index $G$ and mobility $M$ in dependence on two variables: level lines and correlation}

If we compute the values of $G$ in dependence on the two variables $\Delta \tau=\tau_{\max }-\tau_{\min }$ and $\gamma$, we can identify on a cartesian plane $\Delta \tau-\gamma$ a family of "level lines of $G$ " corresponding to constant values of $G$. Similarly, we can find in the same plane the level lines of $M$. In principle, one would expect the two sets of level lines to be distinct. As a matter of fact, it is impossible to write an explicit functional dependence of $G$ and $M$ on $\Delta \tau$ and $\gamma$, and the definitions of the two quantities are completely different. In particular, $G$ depends only on the $\tilde{X}_{i}$, while $M$ also depends on the encounter frequency rates $p_{h k}$, which are largely arbitrary. What is remarkable, however, is that the level lines of $G$ and $M$ coincide up to ca. 1 part in $10^{3}$, at least in the region of the plane $\Delta \tau-\gamma$ which we have explored through the simulations. The increments of $G$ and $M$ are always opposite, so the negative correlation between them is strongly confirmed also in simultaneous dependence on these two variables. Fig. 5 represents, for instance, the three coincident level lines of $G$ and $M$ which start from the points $(\Delta \tau, \gamma)$ equal to $(0.15,0.20),(0.15,0.15)$ and $(0.20,0.15)$. The corresponding values of $\Delta \tau, \gamma, G$ and $M$ are reported in Tab. $1-3$ 


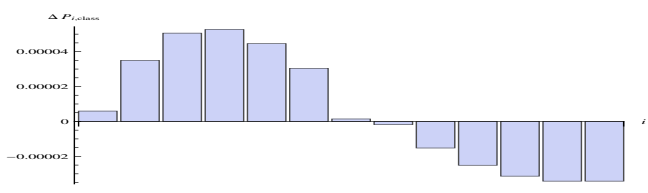

Figure 3: Variation $\Delta P_{i(\text { class })}$ of the class mobility when the tax rates change from $30 \%-45 \%$ to $30 \%-60 \%$, and the Gini index correspondingly decreases from $G=0.368$ to $G=0.338$.

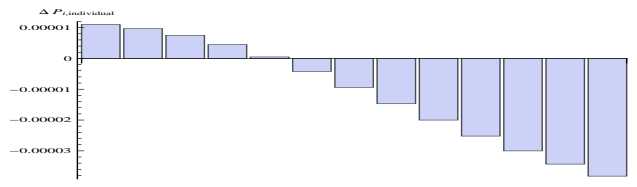

Figure 4: Variation $\Delta P_{i(\text { individual })}$ of the individual mobility when the tax rates change from $30 \%-45 \%$ to $30 \%-60 \%$, and the Gini index correspondingly decreases from $G=0.368$ to $G=0.338$.

\begin{tabular}{ccccc}
\hline$\tau_{\min }-\tau_{\max }$ & $\Delta \tau$ & $\gamma$ & $\mathbf{G}$ & $\mathbf{M}$ \\
\hline $30-45$ & 0.15 & 0.20 & 0.341 & 0.002700 \\
$25-50$ & 0.25 & 0.28 & 0.341 & 0.002704 \\
$20-55$ & 0.35 & 0.36 & 0.341 & 0.002707 \\
\hline
\end{tabular}

Table 1: Data for level line A.

\begin{tabular}{ccccc}
\hline$\tau_{\min }-\tau_{\max }$ & $\Delta \tau$ & $\gamma$ & $\mathbf{G}$ & $\mathbf{M}$ \\
\hline $30-45$ & 0.15 & 0.15 & 0.338 & 0.002712 \\
$25-50$ & 0.25 & 0.24 & 0.338 & 0.002714 \\
$20-55$ & 0.35 & 0.32 & 0.338 & 0.002717 \\
\hline
\end{tabular}

Table 2: Data for level line B.

\begin{tabular}{ccccc}
\hline$\tau_{\min }-\tau_{\max }$ & $\Delta \tau$ & $\gamma$ & $\mathbf{G}$ & $\mathbf{M}$ \\
\hline $27.5-47.5$ & 0.20 & 0.15 & 0.335 & 0.002723 \\
$25-50$ & 0.25 & 0.20 & 0.335 & 0.002723 \\
$20-55$ & 0.35 & 0.28 & 0.335 & 0.002727 \\
\hline
\end{tabular}

Table 3: Data for level line C.

\subsection{Mobility in the kinetic model of a physical gas}

Finally, it is interesting to compare the economic mobility defined and computed in the previous sections with the corresponding intuitive notion applied to a gas. We can base this comparison on the concept of temperature. Independently from its exact definition, mobility should be related to the probability that molecules pass to a state of higher energy after interaction with other molecules. It seems therefore reasonable to assume that mobility increases with absolute temperature. In order to establish a correlation with inequality, we should then determine how the "Gini index of a gas" varies with temperature. For the Boltzmann-Gibbs exponential distribution, the Gini index is independent from the temperature and equal to 0.5. A particular attention is deserved by the $\kappa$-generalized distribution introduced by Kaniadakis [18]. It takes into account relativistic kinematics and is therefore suitable to describe hot gases and plasmas, and at the same time it provides an excellent fit for income distributions both of real world [16] and of kinetic models [13]. The Kanidakis distribution has a Gini index $G$ that increases with temperature. The analytical formula which gives $G$ as a function of the parameters $\alpha$ and $\kappa$ of the distribution contains four Gamma-functions and is given in [17]. When $\alpha=1$ and $\kappa=0$, the distribution reduces to an exponential function with $G=0.5$. For any other value of $\alpha, G$ is an increasing function of $\kappa$ (compare the examples of Fig. 6). When the Kaniadakis distribution is applied 
to a relativistic gas, $\kappa$ is related to the gas parameters by

$$
\frac{1}{\kappa^{2}}=1+\frac{m c^{2}}{k_{B} T},
$$

where $m$ is the atomic or molecular mass, $c$ the speed of light, $k_{B}$ the Boltzmann constant and $T$ the absolute temperature [18]. Eq. [24] shows that $\kappa$ increases when $T$ increases. It follows that a higher temperature leads to higher $G$. This amounts, for the gas, to a negative correlation between equality and mobility, which disagrees with the results of empirical economic studies and of our kinetic model.

This comparison points to one of the many qualitative differences between physical and economical interactions. In physical systems it happens quite often that larger gradients in density, pressure, etc. cause a quicker dynamical evolution, so that, in some sense, "greater inequality causes greater mobility". In economy this "stimulus effect" has been theorized to work for certain growth phases, see [19] and references therein, but is apparently not the dominant factor at equilibrium. Instead, mobility is positively related to equality, at least as an average over all income classes. We have seen that the mobility of the low and middle classes is the key element under this respect.

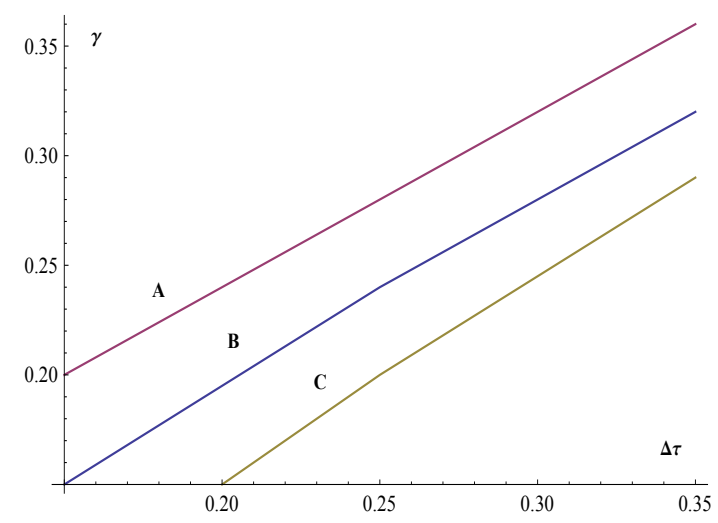

Figure 5: Level lines in the plane $\Delta \tau-\gamma$ corresponding to the following values of Gini index and mobility. A: $G=0.341, M=0.00270$. B: $G=0.338, M=0.00271$. C: $G=0.335, M=0.00272$. Numerical data for the three lines are given resp. in Table $1,2,3$.

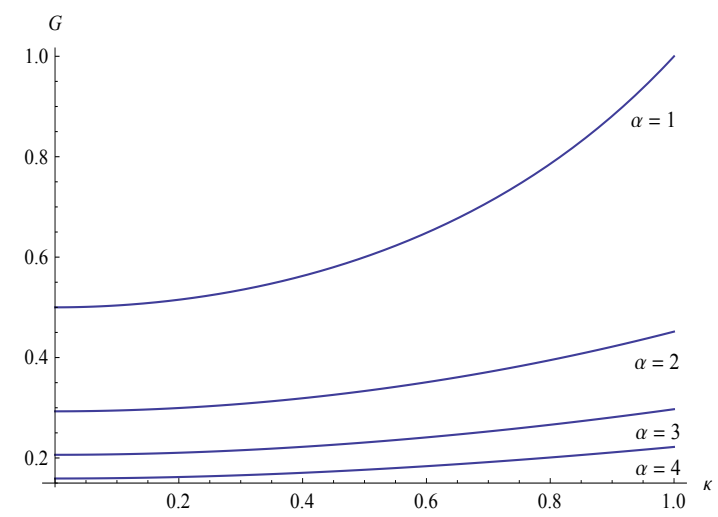

Figure 6: Dependence of the Gini index of the Kaniadakis distribution on the parameters $\alpha$ and $\kappa$, for $\kappa$ in the range $[0,1]$ and the four values $\alpha=1,2,3,4$.

\section{Conclusions}

In this paper microscopic models of economic exchanges, taxation and redistribution, described by kinetic equations are considered. Some features of the emerging income distribution curves are discussed. In particular, we compare 
models with fiscal systems characterized by a different spread between the minimum and the maximum tax rate and models in which the welfare provision is weighted differently for different income classes. The main contribution of the paper is represented, in our opinion, by the introduction of a measure of social mobility. This is possible due to the structure of the equations, which involve several transition probabilities for the passage from an income class to others. Together with the Gini index, this measure serves as a tool to investigate the correlation between economic inequality and social mobility. The correlation turns out to be clearly negative: a lower mobility corresponds to a higher inequality. This is in agreement with what is observed in real world situations. While this negative correlation has been the object of several studies and debates in the socio-economic arena, to our knowledge it has not been treated within the brand of mathematical and physical approaches to complex systems.

\section{References}

[1] Andrews D., Leigh. A., More inequality, less social mobility, Appl. Econ. Lett. 16, 1489-1492 (2009)

[2] Corak M., Income inequality, equality of opportunity, and intergenerational mobility, J. Econ. Perspect. 27, 79-102 (2013)

[3] Kirman A., Complex Economics: Individual and Collective Rationality, Routledge, London (2010)

[4] Gallegati M., Reconstructing economics: Agent Based Models and Complexity, Conference Paper, Berlin, Paradigm Lost: Rethinking economics and Politics. Available at http://ineteconomics.org/conference/berlin/reconstructing-economics-agent-based-models-and-complexity, (2012)

[5] Arthur W.B., Complexity Economics: a different framework for economic thought, in: Arthur W.B., Complexity and the Economy, Oxford University Press, New York (2014)

[6] Tesfatsion L., Judd K.L. (Eds.), Handbook of Computational Economics, Volume 2: Agent-based Computational Economics, North-Holland, Amsterdam (2006)

[7] Dragulescu A., Yakovenko V.M., Statistical mechanics of money, Eur. Phys. J. B, 17, 723-729 (2000)

[8] Sinha S., Chakrabarti B.K., Towards a physics of economics, Physics News (Bullettin of the Indian Physical Association) 39, 33-46 (2009)

[9] Patriarca M., Chakraborti A., Kinetic exchange models: from molecular physics to social science, Am. J. Phys. 81, 618 (2013)

[10] Chatterjee A., Socio-economic inequalities: a statistical physics perspective, arXiv:1409.8030v1 (2014)

[11] Bertotti, M.L., Modelling taxation and redistribution: a discrete active particle kinetic approach. Appl. Math. Comput. 217, 752-762 (2010)

[12] Bertotti, M.L., Modanese G., From microscopic taxation and redistribution models to macroscopic income distributions. Physica A 390, 3782-3793 (2011)

[13] Bertotti, M.L., Modanese G., Exploiting the flexibility of a family of models for taxation and redistribution. Eur. Phys. J. B 85, 261 (2012)

[14] Bertotti, M.L., Modanese G., Micro to macro models for income distribution in the absence and in the presence of tax evasion, Appl. Math. Comput. 244, 836-846 (2014)

[15] Bertotti, M.L., Modanese G., Microscopic models for welfare measures addressing a reduction of economic inequality, submitted (2014)

[16] Clementi F., Di Matteo T., Gallegati M., Kaniadakis G., The א-generalized distribution: a new descriptive model for the size of the distribution of incomes, Physica A 387 3201-3208 (2008)

[17] Clementi F., Gallegati M., Kaniadakis G., A א-generalized statistical mechanics approach to income analysis, J. Stat. Mech. 2009 P02037 (2009)

[18] Kaniadakis G., Statistical mechanics in the context of special relativity, Phys. Rev. E 66, 056125 (2002)

[19] Aghion P., Caroli E., García-Peñalosa C., Inequality and economic growth: the perspective of the new growth theories, J. Econ. Lit. 37, 1615-1660 (1999) 